\title{
Power Level Control of Nuclear Power Plant Based on Asymptotical State Observer under Neutron Sensor Fault
}

\author{
Liming Zhang (iD, Hongyun Xie, Qizhi Duan, Chao Lu, Jixue Li, and Zhihong Lv
}

State Key Laboratory of Nuclear Power Safety Monitoring Technology and Equipment, China Nuclear Power Engineering Co., Ltd., Shenzhen, 518172, China

Correspondence should be addressed to Liming Zhang; zhangliming@cgnpc.com.cn

Received 2 October 2020; Accepted 15 January 2021; Published 3 February 2021

Academic Editor: Leon Cizelj

Copyright $(92021$ Liming Zhang et al. This is an open access article distributed under the Creative Commons Attribution License, which permits unrestricted use, distribution, and reproduction in any medium, provided the original work is properly cited.

Power level control is one of the critical functions in the instrument and control system of nuclear power plants (NPPs). In most power level control systems of NPPs, the power level or average neutron flux in reactor cores provided by out-of-core neutron sensors are usually measured as feedback of power control systems, while, as critical measuring devices, there is a risk of damage to out-of-core neutron sensors. For improving the operation reliability of NPPs under the neutron sensors' failure, a power control system based on power observer is developed in this work. The simulation based on NPP simulator shows the power control system based on the observer is effective when neutron sensors fail.

\section{Introduction}

Ensuring safe and effective operations of nuclear power plants (NPPs) is the precondition during the development [1]. In terms of reactor power control, the implementation of desirable control for a core in an NPP is an important measure to improve security and effectiveness of the core.

In most power control systems of NPPs, the average neutron flux in reactor cores provided by out-of-core neutron sensors is usually measured as feedback of power control systems, which can be shown as Figure 1 [2]. During the past decades, some core power control methods are developed gradually, including optimal control $[3,4]$, neural network [5-7], fuzzy logical control [8], slide mode control $[9,10]$, model predictive control [11-13], and fractional order control $[14,15]$. These control methods have to depend on the neutron flux measurement, while, as critical measuring devices, there is a risk of damage to out-of-core neutron sensors [16]. The neutron sensors are difficult to be replaced and maintained online. If neutron sensors fail, the power control system cannot work normally, and NPPs will be shut down further. On the contrary, the thermocouple in the secondary loop is convenient for replacement and maintenance. Meanwhile, the coolant temperature in secondary can also reflect the core power level. Therefore, it is possible to rebuild the neutron flux and core power level according to the coolant temperature in the secondary loop, when the out-of-core neutron sensors fail.

For improving the operation reliability of NPPs under the neutron sensors' failure, a power control system based on power observer is developed in this work. Firstly, a common NPP dynamic model containing neutron kinetic and thermal-hydraulic is built, and then the formulation of control problem about power level control under neutron sensors' failure is presented; after that, based on the separation principle, the power level observer and power level controller are developed independently; at last, the effectiveness of the power level controller based on the observer is verified.

\section{Modeling}

As shown in Figure 2, the whole plant module of NPPs is mainly composed of a reactor core with thermal neutron, pressurized water, U-tube steam generator (UTSG), main pump, necessary connection pipes, and vessels [17]. Since this study focuses on modeling the dynamic response and sensors fault characteristics between the nuclear reactor 


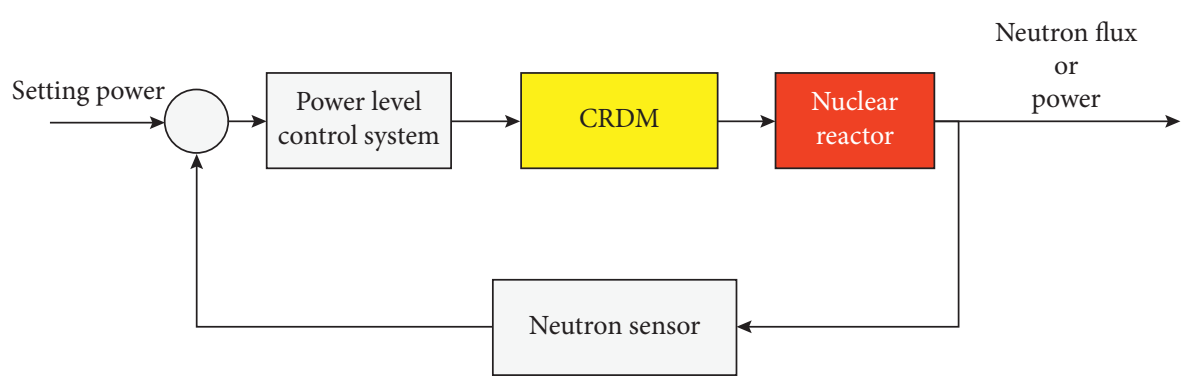

Figure 1: The structure of power level control system in NPP.

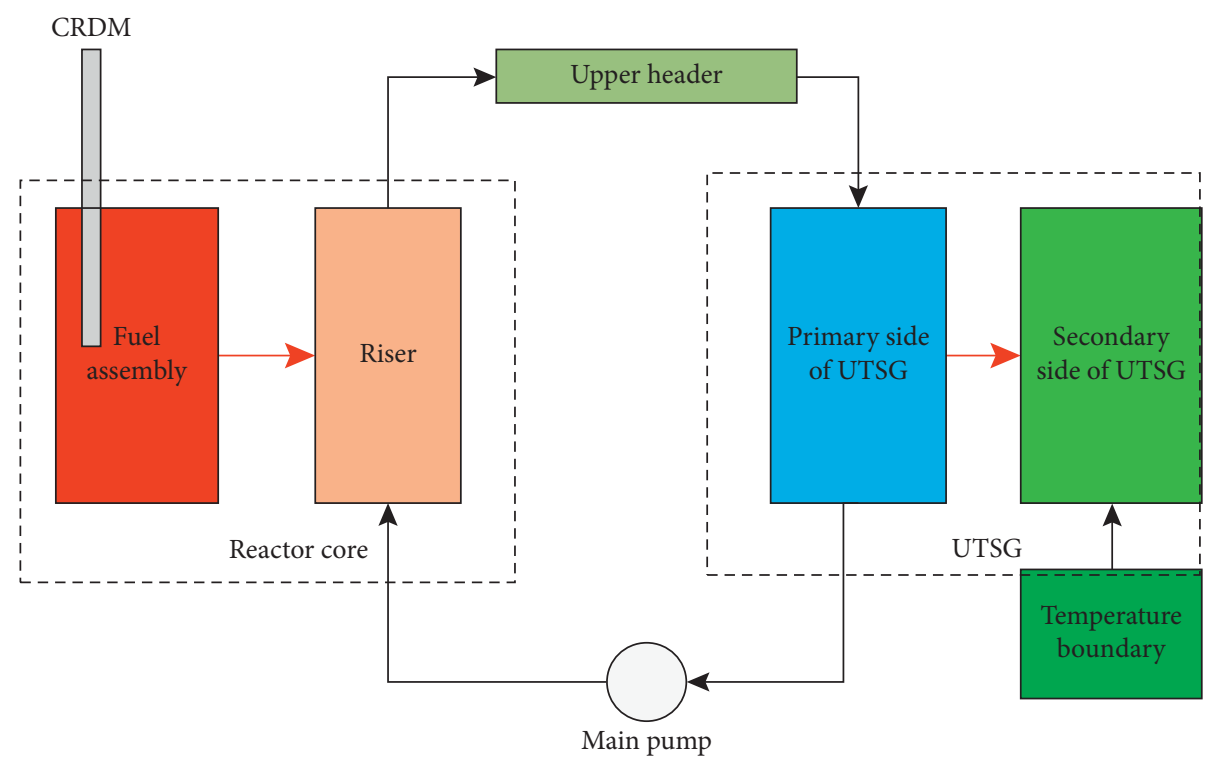

Figure 2: Nodalization scheme of the whole plant module of NPPs.

power and primary coolant as well as proposing a proper control method under the neutron sensors fault to enhance the reliability of the control system, it is reasonable to adopt a simplified model of the lumped parameters core, coolant, and UTSG module, where the corresponding nodalization scheme is shown in Figure 2.
2.1. Neutron Kinetic. According to the lumped parameter method, the nonlinear core model is established via using the point kinetics equations with six groups of delayed neutrons and two reactivity feedback mechanisms [18]. The normalized point kinetics equations for the critical core without external source of neutrons can be expressed as

$$
\frac{\mathrm{d} n(t)}{\mathrm{d} t}=\frac{\delta(t)-\beta}{\Lambda}+\sum_{i=1}^{6} \lambda_{i} c_{i}(t)+q(t), \frac{\mathrm{d} c_{i}(t)}{\mathrm{d} t}=\frac{\beta_{i}}{\Lambda} n(t)-\lambda_{i} c_{i}(t), \quad i=1,2, \ldots, 6
$$

The reactivity feedbacks in this model are expressed as a function of the average temperatures of fuel and coolant. As a result, the total reactivity is the control rod reactivity combined with the feedbacks described as

$$
\delta(t)=\delta_{\text {rod }}+\alpha_{f}\left(T_{f}-T_{f 0}\right)+\alpha_{\text {coolant }}\left(T_{p}-T_{p 0}\right),
$$

where the subscript 0 refers to a reference condition, taken at the rated power, $n$ means the neutron density, $\rho$ means the reactivity, $\beta$ means the neutron fraction, $\Lambda$ means the neutron generation time, $\lambda_{i}$ means the decay constant of $i^{\text {th }}$ group, $c_{i}$ means the delayed neutron precursor concentration of $i^{\text {th }}$ group, $\delta_{\text {rod }}$ means the reactivity of rod, $\alpha_{f}$ means the reactivity feedback of fuel, $\alpha_{\text {coolant }}$ means the reactivity feedback of coolant in reactor core, $T_{f}$ means the temperature of fuel, $T_{f}$ means the temperature of fuel at the rated power, and $T_{p}$ means the average temperature of pressurized water of reactor core.

It is assumed that the neutron density in steady state is $n_{0}$, delayed neutron precursor concentration of $i^{\text {th }}$ group in steady state is $c_{i 0}$, and then the neutron kinetic model can be linearized as follows: 


$$
\frac{\mathrm{d} \Delta n(t)}{\mathrm{d} t}=\frac{\delta_{0}-\beta}{\Lambda} \Delta n(t)+\frac{n_{0}}{\Lambda} \Delta \delta(t)+\sum_{i=1}^{6} \lambda_{i} \Delta c_{i}(t), \frac{\mathrm{d} \Delta c_{i}(t)}{\mathrm{d} t}=\frac{\beta_{i}}{\Lambda} \Delta n(t)-\lambda_{i} \Delta c_{i}(t)
$$

where $\Delta$ means the increment.

$$
\rho_{\text {sgp }} V_{\text {sgp }} C_{p} \frac{\mathrm{d} T_{\text {sgp }}}{\mathrm{d} t}=G_{p} C_{p}\left(T_{\text {sgpin }}-T_{\text {sgpout }}\right)-U_{\text {sg }} A_{\text {sg }} T_{\text {sg } \Delta},
$$

\subsection{Thermal-Hydraulic}

2.2.1. Core. The reactor core is assumed to be a lumped cylindrical area as a function of the power [19]. The fission energy is released in the fuel elements in the form of heat. This heat is then transferred to the upcoming pressurized water. This process is described as follows:

$$
\begin{aligned}
& \rho_{f} V_{f} C_{f} \frac{\mathrm{d} T_{f}}{\mathrm{~d} t}=P-U_{t} A_{c}\left(T_{f}-T_{c}\right), \\
& \rho_{c} V_{c} C_{p} \frac{\mathrm{d} T_{c}}{\mathrm{~d} t}=U_{t} A_{c}\left(T_{f}-T_{c}\right)-G_{p} C_{p}\left(T_{\text {cout }}-T_{\text {cin }}\right),
\end{aligned}
$$

where $\rho_{f}$ and $\rho_{c}$ mean the fuel density and the primary coolant density in the reactor core, respectively, $V_{f}$ means the fuel volume, $P$ means the reactor power, $U_{t}$ means the heat transfer coefficient between fuel and primary coolant, $A_{c}$ means the fuel assemblies area, $C_{f}$ and $C_{p}$ the mean specific enthalpy of fuel and primary coolant, respectively, $T_{f}$ and $T_{c}$ mean fuel and primary coolant temperatures, respectively, and $G_{p}$ means the mass flow rate of primary loop.

Accordingly, the increment form of equations (4) and (5) can be given as

$$
\begin{aligned}
\rho_{f} V_{f} C_{f} \frac{\mathrm{d} \Delta T_{f}}{\mathrm{~d} t} & =\Delta P-U_{t} A_{c}\left(\Delta T_{f}-\Delta T_{c}\right), \\
\rho_{c} V_{c} C_{p} \frac{\mathrm{d} \Delta T_{c}}{\mathrm{~d} t} & \\
= & U_{t} A_{c}\left(\Delta T_{f}-\Delta T_{c}\right) \\
& -G_{p} C_{p}\left(\Delta T_{\text {cout }}-\Delta T_{\text {cin }}\right) .
\end{aligned}
$$

2.2.2. Steam Generator. Usually, steam generators in NPPs will be of U-tubes and counter current type. Single-phase pressurized water and two-phase water are in shell-side and tube-side of USTGs, respectively. In order to give a simple description for the control-oriented modeling, one-section model is adopted to describe the main thermodynamics of UTSGs:

$$
\rho_{\mathrm{sgs}} V_{\mathrm{sgs}} \frac{\mathrm{d} T_{\mathrm{sgs}}}{\mathrm{d} t}=G_{s} C_{s}\left(T_{\mathrm{fw}}-T_{\text {sgsout }}\right)+U_{\mathrm{sg}} A_{\mathrm{sg}} T_{\mathrm{sg} \Delta},
$$

where $T_{\text {sgp }}$ means the average coolant temperature in the primary side of UTSGs, $T_{\text {sgpin }}$ and $T_{\text {sgpout }}$ mean inlet and outlet coolant temperatures in the primary side of UTSGs, $T_{\text {sg } \Delta}$ means the temperature difference between the coolant flows in the two sides of UTSGs, which can be given by $T_{\text {sg } \Delta}=T_{\text {sgp }}-T_{\text {sgs }}$, where $T_{\text {sgp }}$ and $T_{\text {sgs }}$ mean average coolant temperatures in primary and secondary sides of UTSGs, respectively, $T_{\mathrm{fw}}$ means the feedwater temperature, $T_{\text {sgsout }}$ means the outlet temperature in secondary side of UTSGs, $U_{\text {sg }}$ and $A_{\text {sg }}$ mean the heat transfer coefficient and area between the two sides of UTSGs, respectively, and $V_{\text {sgp }}$ and $V_{\text {sgs }}$ mean primary and secondary side volume of UTSGs, respectively.

Accordingly, the increment form of equations (7) and (8) can be given as

$$
\begin{gathered}
\rho_{\text {sgp }} V_{\text {sgp }} C_{p} \frac{\mathrm{d} \Delta T_{\text {sgp }}}{\mathrm{d} t}=G_{p} C_{p}\left(\Delta T_{\text {sgpin }}-\Delta T_{\text {sgpout }}\right)-U_{\text {sg }} A_{\mathrm{sg}} \Delta T_{\mathrm{sg} \Delta}, \\
\rho_{\mathrm{sgs}} V_{\mathrm{sgs}} C_{s} \frac{\mathrm{d} \Delta T_{\mathrm{sgs}}}{\mathrm{d} t}=G_{s} C_{s}\left(\Delta T_{\mathrm{fw}}-\Delta T_{\text {sgsout }}\right)+U_{\mathrm{sg}} A_{\mathrm{sg}} \Delta T_{\mathrm{sg} \Delta} .
\end{gathered}
$$

2.3. Coupling Process. In the NPP model, $(\Delta n, \Delta P)$, $\left(\Delta T_{\text {cout }}, \Delta T_{\text {sgpin }}\right)$, and $\left(\Delta T_{\text {sgpout }}, \Delta T_{\text {cin }}\right)$ are three pairs of couple variables:

$$
\begin{aligned}
\Delta P & =\frac{\sum_{f} \Delta n U_{e} \mathrm{~B}}{3.125 \times 10^{10}} \Delta n, \\
\Delta T_{\text {sgpin }}+\tau_{1} \frac{\mathrm{d} \Delta T_{\text {sgpin }}}{\mathrm{d} t} & =\Delta T_{\text {cout }}, \\
\Delta T_{\text {cin }}+\tau_{2} \frac{\mathrm{d} \Delta T_{\text {cin }}}{\mathrm{d} t} & =\Delta T_{\text {sgpout }},
\end{aligned}
$$

where $\tau_{1}$ is the time delay from reactor core outlet to UTSG inlet, and $\tau_{2}$ is the time delay from UTSG outlet to reactor inlet. 
If the control rod reactivity and feedwater temperature are regarded as system input, the coupling process of the NPPs can be shown as Figure 3, and the NPP model can be converted to state space (11):

$$
\frac{\mathrm{d} x}{\mathrm{~d} t}=\mathbf{A} x+\mathbf{B} u
$$

where

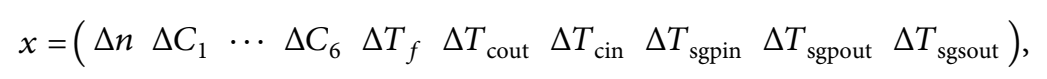

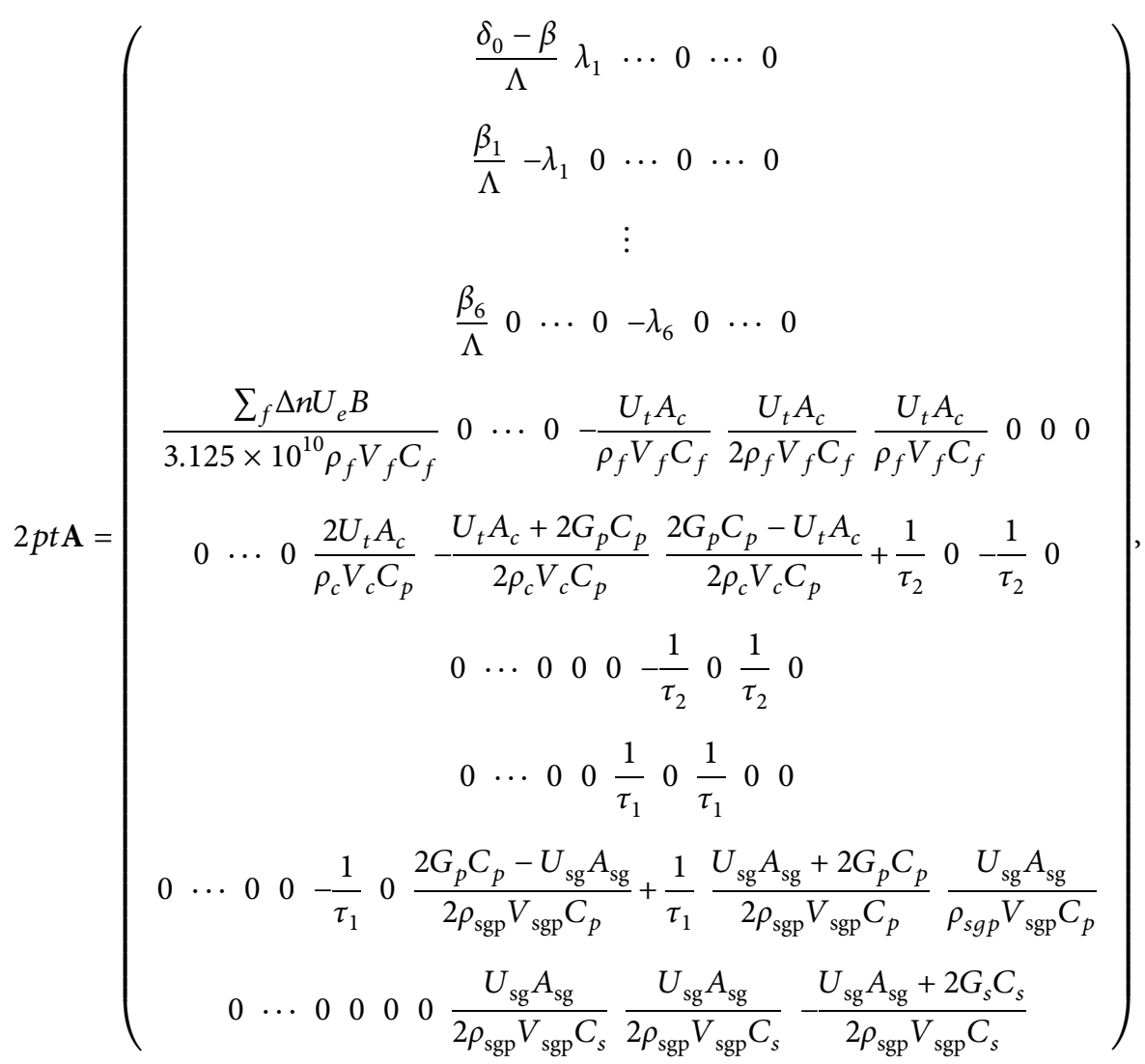

$$
\begin{aligned}
& B=\left(\begin{array}{llll}
\frac{n_{0}}{\Lambda} & 0 & \ldots & 0
\end{array}\right)^{T} \\
& C=\left(\begin{array}{llll}
\frac{\Sigma_{f} \Delta n U_{e} \mathrm{~B}}{3.125 \times 10^{10}} & 0 & \cdots & 0
\end{array}\right) .
\end{aligned}
$$

\section{Analysis and Design of Control System}

3.1. Formulation of Control Problem. Generally, the full state feedback can be used for the whole plant control system. However, in this work, it is assumed that the control system cannot depend on neutron flux feedback from neutron sensors in core or out of core, so that, as a part of state feedback, the nuclear core power should be reconstructed by the output state. As mentioned in Section 1, the accuracy of temperature sensors in the secondary loop can be guaranteed, because maintenance and replacement of temperature sensors in the secondary loop are more convenient than the temperature sensors in primary loop. So, the outlet temperature increment is regarded as the output variable of system (11), as shown as the following equation:

$$
y=\mathbf{C} x
$$

where
The control problem in this work can be given as follows: For system (11) and output (14), design a control system to make the state $x$ converge to 0 , where $\Delta n$ or $\Delta P$ cannot be measured.

3.2. Observer Design. Accordingly, the observer of core power and core temperature can be given as follows:

$$
\widehat{x}=\mathbf{O}(y) \text {. }
$$

In fact, equation (17) is an open loop observer. There are two disadvantages for this observer:

(1) The initial state $x_{0}$ is necessary

(2) If $\hat{x}_{0}$ or $x_{0}$ has deviation caused by system perturbation, the error $\widetilde{e} \triangleq x-\widehat{x}$ will be unstable because 


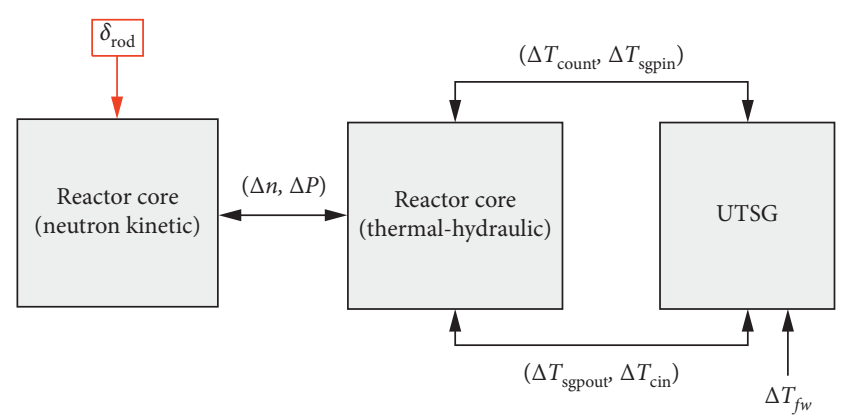

Figure 3: Coupling process of the NPPs.

the state matrix $\mathbf{A}$ has eigenvalues with a positive real part

To improve observation performance, the closed loop observer needs to be built. Accordingly, the closed loop observer model can be given as follows [20]:

$$
\dot{\hat{x}}=(\mathbf{A}-\mathbf{L C}) \hat{x}+\mathbf{L} y+\mathbf{B} u \text {. }
$$

The observation of equation (17) is asymptotically stable. $\hat{x}$ can be regarded as the estimate of $x$ at any time. Actually, the key problem is the eigenvalues configuration of the matrix $(\mathbf{A}-\mathbf{L C})$. The system $\left\{\mathbf{A}^{\mathbf{T}} \mathbf{C}^{\mathbf{T}}\right\}$ is controllable if the system $\{\mathbf{A} \mathbf{C}\}$ is observable. Further, it can be shown that eigenvalues of $\left(\mathbf{A}^{\mathbf{T}}+\mathbf{C}^{\mathrm{T}} \mathbf{K}\right)$ can be configured arbitrarily. If $\mathbf{L}=-\mathbf{K}^{T}$, one has

$$
\mathbf{A}+\mathbf{K}^{\mathrm{T}} \mathbf{C}=\mathbf{A}-\mathbf{L C},
$$

where $\mathbf{K}$ means the state feedback matrix.

It is obvious that eigenvalues of $(\mathbf{A}-\mathbf{L C})$ can also be configured arbitrarily.

3.3. Controller Design. The controller designed in this work needs to depend on the estimate $\hat{x}$. The system with asymptotical state observer (17) can be rewritten as follows:

$$
\left(\begin{array}{c}
\dot{x} \\
\dot{\hat{x}}
\end{array}\right)=\left(\begin{array}{cc}
\mathbf{A} & \mathbf{B K} \\
\mathbf{L C} & \mathbf{A}+\mathbf{B K}-\mathbf{L C}
\end{array}\right)\left(\begin{array}{c}
x \\
e \dot{\hat{x}}
\end{array}\right)+\left(\begin{array}{c}
\mathbf{B} \\
\mathbf{B}
\end{array}\right) v .
$$
has

Under the linear transformation $\left(\begin{array}{c}\dot{x} \\ \dot{e}\end{array}\right)=\mathbf{T}\left(\begin{array}{c}\dot{x} \\ \dot{\hat{x}}\end{array}\right)$, one

$$
\begin{aligned}
\left(\begin{array}{c}
\dot{x} \\
\dot{e}
\end{array}\right) & =\left(\begin{array}{cc}
\mathbf{A}+\mathbf{B K} & -\mathbf{B K} \\
0 & \mathbf{A}-\mathbf{L C}
\end{array}\right)\left(\begin{array}{l}
x \\
e
\end{array}\right)+\left(\begin{array}{l}
\mathbf{B} \\
0
\end{array}\right) v, \\
y & =\left(\begin{array}{ll}
\mathbf{C} & 0
\end{array}\right)\left(\begin{array}{l}
x \\
e
\end{array}\right),
\end{aligned}
$$

where

$$
\mathbf{T}=\left(\begin{array}{cc}
\mathbf{I}_{\mathbf{n}} & 0 \\
\mathbf{I}_{\mathbf{n}} & -\mathbf{I}_{\mathbf{n}}
\end{array}\right) .
$$

It is obvious that $\mathbf{T}$ cannot change feature frequencies, so that the feature spectrums of (20) and (21) can be given as

$$
\sigma A^{\prime}=\sigma(\mathbf{A}+\mathbf{B K}) \oplus \sigma(\mathbf{A}-\mathbf{L} \mathbf{C})
$$

where

$$
A^{\prime}=\left(\begin{array}{cc}
\mathbf{A} & \mathbf{B K} \\
\mathbf{L C} & \mathbf{A}+\mathbf{B K}-\mathbf{L C}
\end{array}\right) .
$$

According to equation (24), system (21) consists of a closed loop subsystem with state feedback $x$ and an estimate subsystem. If the system $\{\mathbf{A} \mathbf{C}\}$ is observable, the feature spectrum $\sigma(\mathbf{A}-\mathbf{L C})$ of the estimate subsystem can be

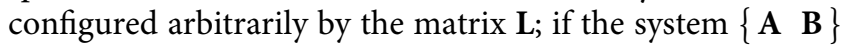
is controllable, the feature spectrum $\sigma(\mathbf{A}+\mathbf{B K})$ can also be configured arbitrarily by the matrix $\mathbf{K}$.

Because of the characteristic of separation design, the feedback matrix can be designed according to the algorithm of pole assignment:

(1) If $\left(\begin{array}{lll}\mathbf{A}_{c} & \mathbf{B}_{c} & \mathbf{C}_{c}\end{array}\right)$ is the controllable form of system (11), calculate the characteristic polynomial $P(s)=$ $\prod_{i=1}^{13}\left(s-p_{i}\right)=s^{13}+\sum_{i=0}^{12} \alpha_{i} s^{i}$ of $\left(\begin{array}{lll}\mathbf{A}_{c} & \mathbf{B}_{c} & \mathbf{C}_{c}\end{array}\right)$, where $p_{i}$ means the $i^{\text {th }}$ pole

(2) If the idea poles are $\overline{p_{i}}, i=1, \ldots, 13$, calculate the polynomial $\overline{P(s)}=\prod_{i=1}^{13}\left(s-\overline{p_{i}}\right)$

(3) The feedback matrix $\mathbf{K}$ can be given as

$$
\mathbf{K}=\boldsymbol{\alpha}-\overline{\mathbf{\alpha}},
$$

where $\alpha=\left(\begin{array}{lll}\alpha_{0} & \ldots & \alpha_{12}\end{array}\right), \bar{\alpha}=\left(\begin{array}{lll}\overline{\alpha_{0}} & \ldots & \overline{\alpha_{12}}\end{array}\right)$, and

$$
\overline{\alpha_{i}}=\left.\frac{1}{i !} \frac{\mathrm{d}^{i} \overline{P(s)}}{\mathrm{d} t^{i}}\right|_{s=0} \text {. }
$$

\section{Results and Discussion}

4.1. Reference Design. To verify the efficiency of the control strategy and the controller in Section 3, the CPR1000 is regarded as the reference design.

CPR1000 (Chinese pressurized reactor) is a three-loop $1000 \mathrm{MWe}$ pressurized water reactor (PWR). It is an advanced, mature, safe, and economical nuclear reactor which is an updated version of Daya Bay Nuclear Reactor. The CPR1000 utilizes the proven technology, which builds on nearly 40 years of operating PWR experience. The major parameters of CPR1000 are listed in Table 1 [21].

Meanwhile, the simulator with CPR1000 type is used for accurate dynamic simulation. The structure of CPR1000 simulation model is shown schematically in Figure 4. For ease of the verification, three components of the model structure are extracted: the reactor core, primary loop, and USTG. The steam turbine, condenser, third loop, and other auxiliary components are instead of temperature and pressure boundaries.

4.2. Poles Distribution. According to the control strategy in Section 3.1, the closed loop poles without the controller in Section 3.2 can be given as Figure 5. It is obvious that the system is not stable, because there are a pair of complex poles in the right half of the plane $(20.8 \pm 25.5 i)$, so that we should reconfigure the unstable poles according to the method in 
TABLE 1: Major parameters of CPR1000.

\begin{tabular}{lc}
\hline Parameters & Value \\
\hline Reactor type & $\mathrm{PWR}$ \\
Fuel type & $\mathrm{UO}_{2}$ \\
Core power & $2895 \mathrm{MW}$ (thermal) \\
Steam generator & $\mathrm{U}$-tube natural circulation \\
Primary pump & Nonseal centrifugal pump \\
Operating pressure & $15.5 \mathrm{MPa}$ \\
Core inlet temperature & $292.4^{\circ} \mathrm{C}$ \\
Core outlet temperature & $327.6^{\circ} \mathrm{C}$ \\
Design volume flow flux & $22840\left(\mathrm{~m}^{3} / \mathrm{h}\right)$ \\
\hline
\end{tabular}

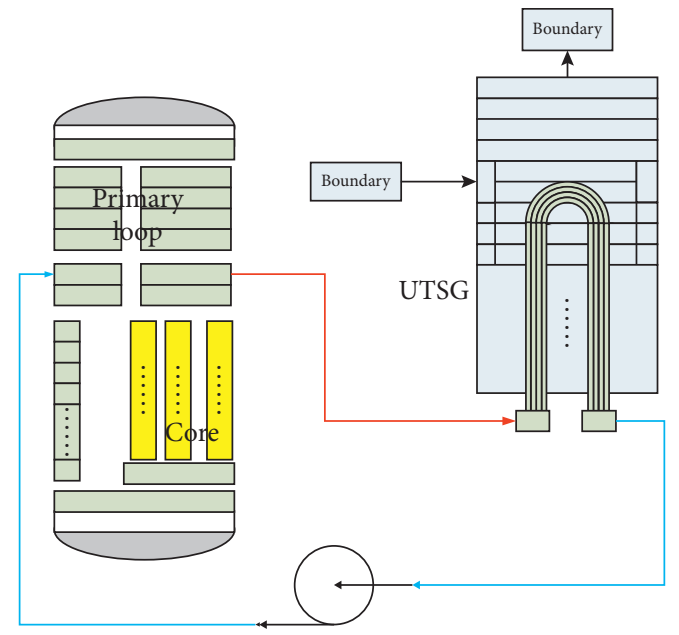

FIGURE 4: CPR1000 simulation model.

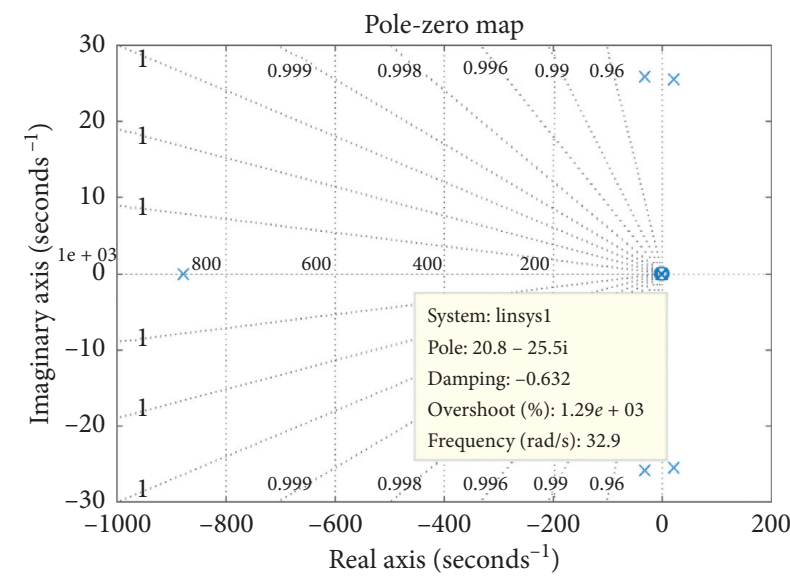

Figure 5: Closed loop pole distribution without the controller in Section 3.3.

Section 3.2. The pair of complex poles is reset at $0.22 \pm 0.47 i$, which can be shown in Figure 6. Meanwhile, three pairs of complex poles, $0.22 \pm 0.47 i, \quad 0.324 \pm 0.00836 i$, and $0.361 \pm 0.101 i$, become the predominant poles. Accordingly, the feedback matrix can be given according to equation (26). Furthermore, the observer of core power can also be designed according to equation (18).

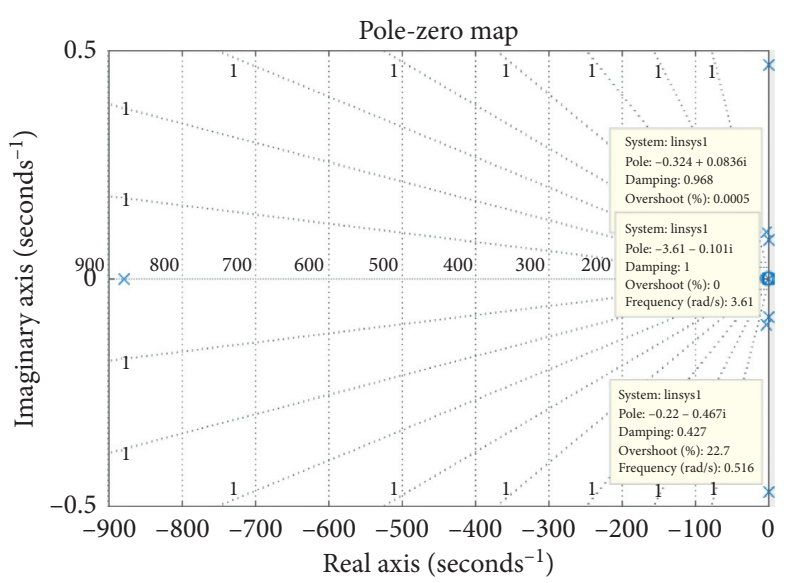

Figure 6: Closed loop pole distribution with the controller in Section 3.3.

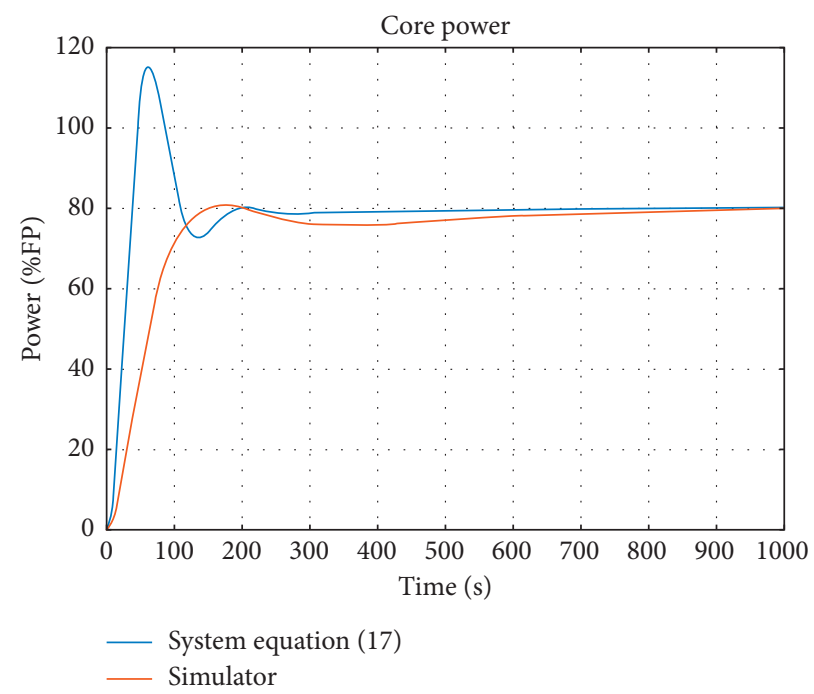

FIgURe 7: Power level response of linear model equation (11) and CPR1000 simulator.

4.3. Dynamic Response. For verifying the effectiveness clearly, two dynamic responses are calculated: one is the response of the linear model equation (11), and the other is the response of the model in the CPR1000 simulator.

In the initial condition, the core power is at $80 \% \mathrm{FP}$, and the mass flow of primary loop is kept constant. At the $1^{\text {st }} \mathrm{sec}$, the setting power is changed from $80 \% \mathrm{FP}$ to $100 \% \mathrm{FP}$. It is assumed that the out-of-core neutron sensors fail completely. The neutron flux feedback can be given by the observer equation (18), where the input contains core outlet coolant temperature and UTSG outlet coolant temperature in secondary side. The dynamic response of core power, core outlet temperature increment, and UTSG outlet temperature increment can be shown as Figures $7-9$. Before $100^{\text {th }} \mathrm{sec}$, the core power and temperature increment dynamic response of system equation (11) have an overshoot of less than $10 \%$ and $5 \%$, respectively. After $400^{\text {th }} \mathrm{sec}$, the core power and temperature increment tend towards stability. In comparison, the power and temperature increment dynamic responses 


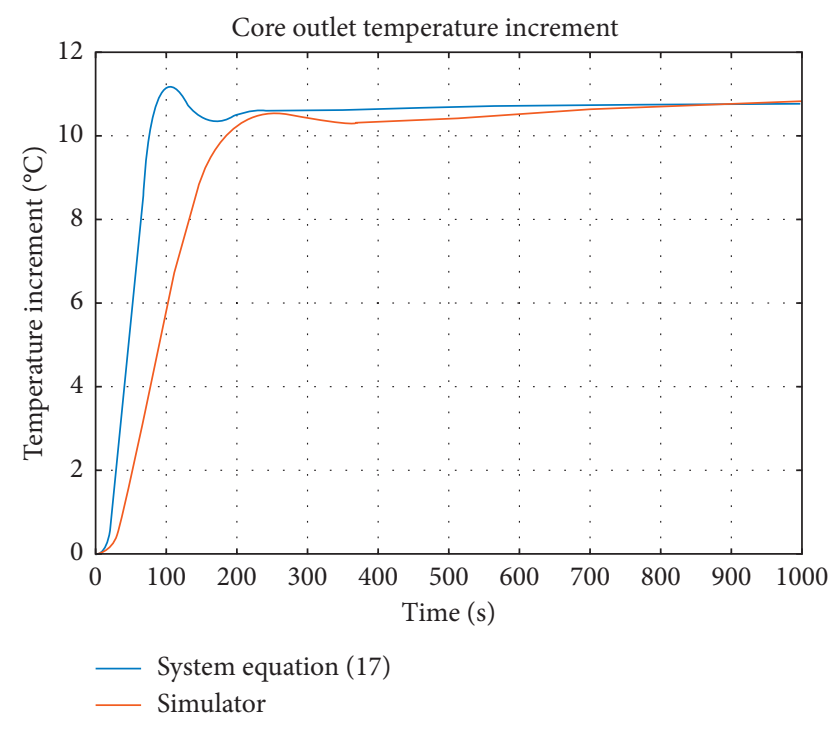

Figure 8: Core outlet temperature response of linear model equation (11) and CPR1000 simulator.

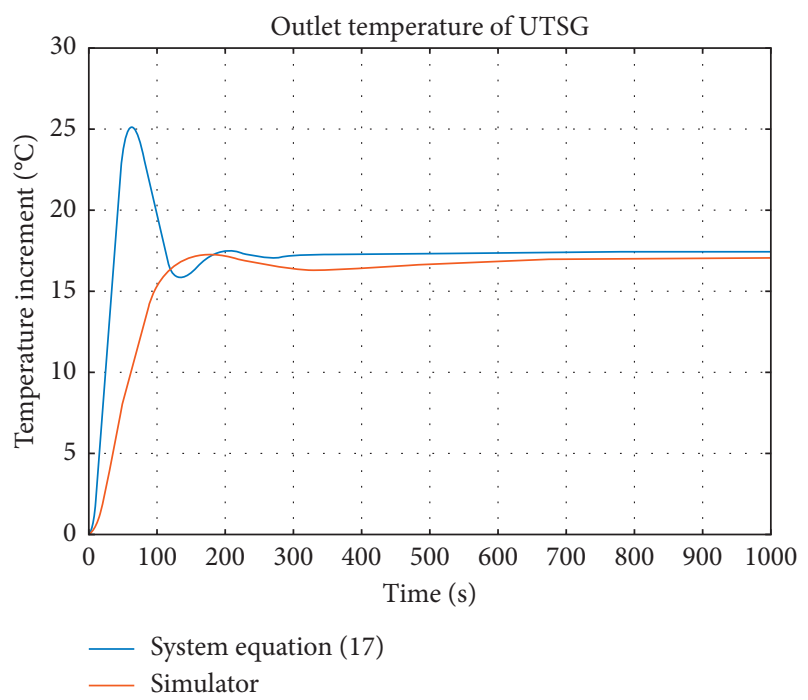

FIGURE 9: UTSG outlet temperature response of linear model equation (11) and Yangjiang NPP simulator.

have same steady state although that they have longer response time. This is due to that some high-frequency components in the simulator are neglected in the linear model equation (11).

\section{Conclusion}

In this work, a power control system of NPPs based on power observer is developed. When the out-of-core neutron sensors fail, the traditional power level control system cannot work because accurate neutron flux and total power in the reactor core cannot be provided. However, because of the separation principle, the neutron flux or total power in the reactor core can be observed by the coolant temperature in the secondary loop and independent from power controller design, so that the state observer and controller are designed, respectively, and the effectiveness of the power level controller based on the state observer is verified by the NPP simulator. The analysis shows consistency of results between the linear model and NPP simulator, which indicates that the power level controller based on the state observer is effective, although there is difference in the dynamic response, which is caused by the truncation of the high frequencies.

In the future work, we will focus on the nonlinear core power observer and eliminate the dynamic deviation caused by the truncation for high frequencies.

\section{Data Availability}

The nuclear data used to support the findings of this study have not been made available because the limit of the law in China.

\section{Conflicts of Interest}

The authors declare that they have no conflicts of interest.

\section{Acknowledgments}

The authors thank the great help from Southeast University in this research. This work was supported in part by the Guangdong Basic and Applied Basic Research Foundation (project no. 2019B1515120060) and in part by the Open Funds of State Key Laboratory of Nuclear Power Safety Monitoring Technology and Equipment.

\section{References}

[1] G. Li, X. Wang, B. Liang, X. Li, B. Zhang, and Y. Zou, "Modeling and control of nuclear reactor cores for electricity generation: a review of advanced technologies," Renewable and Sustainable Energy Reviews, vol. 60, pp. 116-128, 2016.

[2] G. Ablay, "A modeling and control approach to advanced nuclear power plants with gas turbines," Energy Conversion and Management, vol. 76, pp. 899-909, 2013.

[3] F. Zhao, K. C. Cheung, and R. M. K. Yeung, "Optimal power control system of a research nuclear reactor," Nuclear Engineering and Design, vol. 219, no. 3, pp. 247-252, 2003.

[4] M. G. Park and N. Z. Cho, "Time-optimal control of nuclearreactor power with adaptive proportional integral feedforward gains," Institute of Electrical and Electronics Engineers Transactions on Nuclear Science, vol. 40, no. 3, pp. 266-270, 1993.

[5] C.-C. Ku, K. Y. Lee, and R. M. Edwards, "Improved nuclear reactor temperature control using diagonal recurrent neural networks," Institute of Electrical and Electronics Engineers Transactions on Nuclear Science, vol. 39, no. 6, pp. 2298-2308, 1992.

[6] M. N. Khajavi, M. B. Menhaj, and A. A. Suratgar, "A neural network controller for load following operation of nuclear reactors," Annals of Nuclear Energy, vol. 29, no. 6, pp. 751-760, 2002.

[7] S. S. Khorramabadi, M. Boroushaki, and C. Lucas, "Emotional learning based intelligent controller for a PWR nuclear reactor core during load following operation," Annals of $\mathrm{Nu}$ clear Energy, vol. 35, no. 11, pp. 2051-2058, 2008. 
[8] E. Rojas-Ramírez, J. S. Benítez-Read, and A. Segovia-De-Los Ríos, "A stable adaptive fuzzy control scheme for tracking an optimal power profile in a research nuclear reactor," Annals of Nuclear Energy, vol. 58, pp. 238-245, 2013.

[9] R. K. Munje, B. M. Patre, S. R. Shimjith, and A. P. Tiwari, "Sliding mode control for spatial stabilization of advanced heavy water reactor," Institute of Electrical and Electronics Engineers Transactions on Nuclear Science, vol. 60, no. 4, pp. 3040-3050, 2013.

[10] G. R. Ansarifar and H. R. Akhavan, "Sliding mode control design for a PWR nuclear reactor using sliding mode observer during load following operation," Annals of Nuclear Energy, vol. 75, pp. 611-619, 2015.

[11] A. Etchepareborda and J. Lolich, "Research reactor power controller design using an output feedback nonlinear receding horizon control method," Nuclear Engineering and Design, vol. 237, no. 3, pp. 268-276, 2007.

[12] H. Eliasi, M. B. Menhaj, and H. Davilu, "Robust nonlinear model predictive control for nuclear power plants in load following operations with bounded xenon oscillations," $\mathrm{Nu}$ clear Engineering and Design, vol. 241, no. 2, pp. 533-543, 2011.

[13] Y. Tai, S. X. Hou, C. Li, and F. Y. Zhao, “An improved implicit multiple model predictive control used for movable nuclear power plant," Nuclear Engineering and Design, vol. 240, no. 10, pp. 3582-3585, 2010.

[14] S. S. Ray and A. Patra, "Numerical simulation for fractional order stationary neutron transport equation using Haar wavelet collocation method," Nuclear Engineering and Design, vol. 278, pp. 71-85, 2014.

[15] T. K. Nowak, K. Duzinkiewicz, and R. Piotrowski, "Numerical solution analysis of fractional point kinetics and heat exchange in nuclear reactor," Nuclear Engineering and Design, vol. 281, pp. 121-130, 2015.

[16] S. Shimjith, A. Tiwari, and B. Bandyopadhyay, "Multipoint kinetics modeling of large nuclear reactors," in Modeling and Control of a Large Nuclear Reactor: A Three-Time-Scale Approach, S. R. Shimjith, A. P. Tiwari, and B. Bandyopadhyay, Eds., pp. 17-60, Springer Berlin Heidelberg, Heidelberg, Germany, 2013.

[17] S. Yan, H. Fang, P. Wang et al., "Modeling and control strategy of the China accelerator driven subcritical reactor," Progress in Nuclear Energy, vol. 71, pp. 179-187, 2014.

[18] M. Colombo, A. Cammi, V. Memoli, D. Papini, and M. E. Ricotti, "Transfer function modelling of the lead-cooled fast reactor (LFR) dynamics," Progress in Nuclear Energy, vol. 52, no. 8, pp. 715-729, 2010.

[19] Z. Dong, Y. Pan, Z. Zhang, Y. Dong, and X. Huang, "Dynamical modeling and simulation of the six-modular high temperature gas-cooled reactor plant HTR-PM600," Energy, vol. 155, pp. 971-991, 2018.

[20] F. M. Callier, C. A. D. Controllability, and Observability, Linear System Theory, pp. 222-264, Springer New York, New York, NY, USA, 1991.

[21] S. Wu, P. Wang, H. Song, X. Wei, F. Zhao, and S. Revankar, "Modeling and load follow simulation of CPR1000 Nuclear Power Plant implementing Mechanical Shim control strategy," Nuclear Engineering and Design, vol. 352, Article ID 110161, 2019. 